\title{
DEVELOPMENT OF THE PETAL LASER FACILITY AND ITS DIAGNOSTIC TOOLS
}

\author{
Dimitri Batani ${ }^{a, *}$, Sebastien $\operatorname{Hulin}^{a}$, Jean Eric Ducret $^{a}$, \\ Emmanuel D'Humières ${ }^{a}$, Vladimir TikhonchuK $^{a}$, JÉrôme Caron $^{a}$, \\ Jean-Luc Feugeas $^{a}$, Philippe Nicolai $^{a}$, Michel Koenig $^{b}$, \\ Serena Bastiani-Ceccotti ${ }^{b}$, Julien Fuchs $^{b}$, Tiberio Ceccottio ${ }^{c}$, \\ Sandrine Dobosz-Dufrenoy ${ }^{c}$, Cecile Szabo-Foster $^{d}$, Laurent Serani ${ }^{e}$, \\ Luca Volpe ${ }^{f}$, Claudio Perego $^{f}$, Isabelle Lantuejoul-Thfoin ${ }^{g}$, \\ Eric Lefèbvre ${ }^{g}$, Antoine Compant La Fontaine ${ }^{g}$, Jean-Luc Miquel ${ }^{g}$, \\ Nathalie Blanchot ${ }^{g}$, Alexis Casner ${ }^{g}$, Alain Duval ${ }^{g}$, Charles Reverdin ${ }^{g}$, \\ René Wrobel ${ }^{g}$, Julien Gazave ${ }^{g}$, Jean-Luc Dubois ${ }^{g}$, Didier Raffestin ${ }^{g}$ \\ ${ }^{a}$ Université Bordeaux, CEA, CNRS, CELIA UMR 510\%, F-33400 Talence, France \\ ${ }^{b}$ LULI, UMR 7605, Ecole Polytechnique, F-91128 Palaiseau, France \\ ${ }^{c}$ IRAMIS/Service Photons Atomes et Molecules, CEA-Saclay, F-91191 Gif sur Yvette, France \\ ${ }^{d}$ Laboratoire Kastler-Brossel UMR 8552, 4, place Jussieu, F-75252 Paris, France \\ e CENBG UMR 5797, Chemin du Solarium, F-33175 Gradignan, France \\ $f$ Universitá degli Studi di Milano-Bicocca, I-20126 Milan, Italy \\ g CEA-CESTA, BP 2, F-33114 Le Barp, France \\ * corresponding author: batani@celia.u-bordeaux1.fr
}

Abstract. The PETAL system (PETawatt Aquitaine Laser) is a high-energy short-pulse laser, currently in an advanced construction phase, to be combined with the French Mega-Joule Laser (LMJ). In a first operational phase (beginning in 2015 and 2016) PETAL will provide $1 \mathrm{~kJ}$ in $1 \mathrm{ps}$ and will be coupled to the first four LMJ quads. The ultimate performance goal to reach $7 \mathrm{PW}(3.5 \mathrm{~kJ}$ with $0.5 \mathrm{ps}$ pulses). Once in operation, LMJ and PETAL will form a unique facility in Europe for High Energy Density Physics (HEDP). PETAL is aiming at providing secondary sources of particles and radiation to diagnose the HED plasmas generated by the LMJ beams. It also will be used to create HED states by short-pulse heating of matter.

Petal+ is an auxiliary project addressed to design and build diagnostics for experiments with PETAL. Within this project, three types of diagnostics are planned: a proton spectrometer, an electron spectrometer and a large-range $\mathrm{X}$-ray spectrometer.

KEYWORDS: plasma diagnostic, X-ray photon emission, proton radiography, particle laser acceleration, Laser MegaJoule, Petawatt laser, High Energy Density Physics, Electron spectrometer, X-ray spectrometer.

\section{INTRODUCTION}

A new era of plasma science started with the first experiments on the National Ignition Facility (NIF) at the Lawrence Livermore National Laboratory (LLNL) in the USA. Up to now, the 192 beams of the NIF have been able to deliver more than 1.8 MJ of energy into a hohlraum target. The aim is to reach the target ignition by indirect drive, the laser energy being transformed into a high-intensity and hightemperature radiation field, which is irradiating and compressing the target [11, 15].

The Laser MegaJoule (LMJ) under construction near Bordeaux in France is following the trail opened by the NIF with its planned 160 laser beams for more than $1 \mathrm{MJ}$ to reach ignition of a deuterium-tritium target using the indirect drive method. An updated status report on the LMJ was made at the IFSA (Inertial Fusion Science \& Applications) conference, held in Bordeaux in September 2011 [19] (MIQ11). The construction plan leads to the beginning of laser shots on LMJ at the end of 2014. The laser lines of the LMJ will be assembled in quads of 4 beamlets. Each quad will deliver more than $30 \mathrm{~kJ}$ of energy within a few ns, providing an intensity of about $10^{15} \mathrm{~W} \mathrm{~cm}^{-2}$. At the start of the operation, four quads will be available with the first LMJ-PETAL experiments for the academic science community in mid-2015.

Besides the physics of ICF (plasma physics, shock/fast ignition), NIF \& LMJ will be essential for basic science, exploring fields such as plasma astrophysics (e.g. study of shocks to simulate violent events 


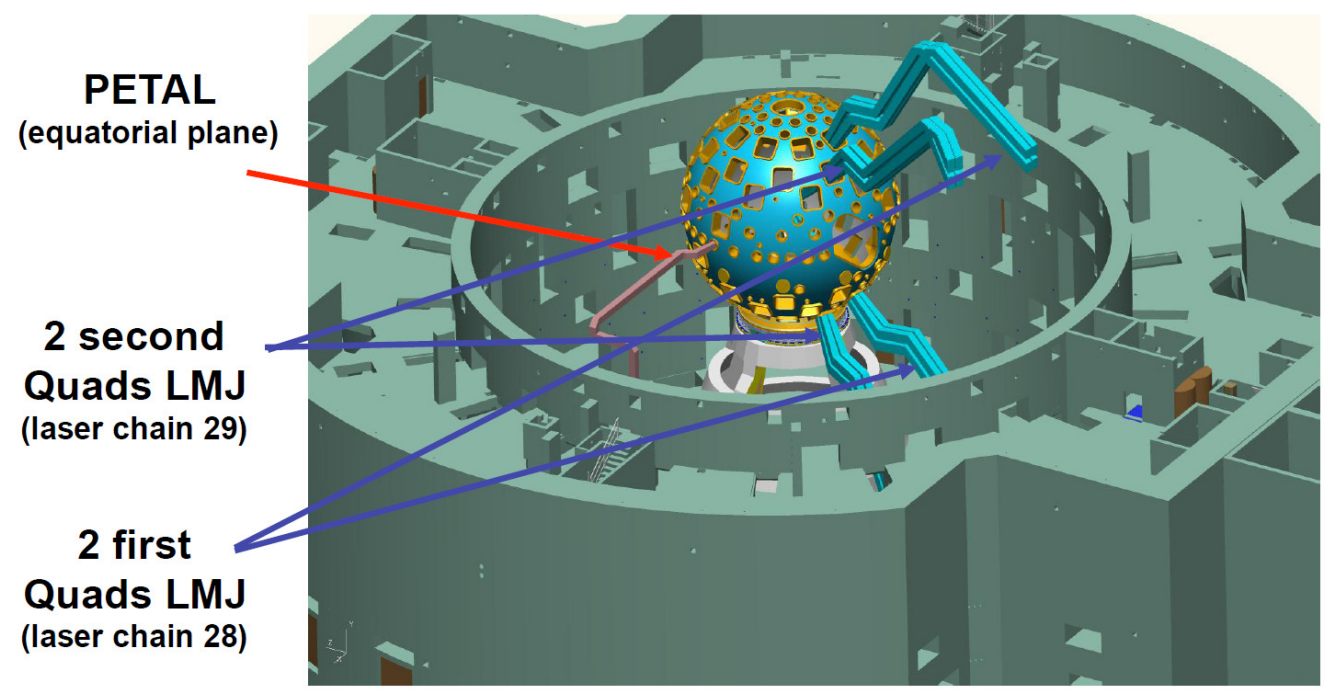

FIgURE 1. Initial configuration of the LMJ/PETAL laser system.

in the Universe such as supernovae, accretion disks), planetary physics (highly compressed and warm matter), stellar interiors with large coupling between radiation field and matter and nuclear physics. Overviews of the physics programme at LMJ and NIF may be found in [16, 20].

A PW short pulse laser will be added to the nanosecond pulse beams of the LMJ. This is the PETAL system, under construction on the LMJ site near Bordeaux (France). It is supported and funded by the Region Aquitaine Regional Council.

Once in operation, LMJ \& PETAL will be a unique facility in Europe for High Energy Density Physics (HEDP).

\section{PETAL LASER SYSTEM}

The PETAL system, under construction on the LMJ site, has the ultimate goal to reach $7 \mathrm{PW}(3.5 \mathrm{~kJ}$ with $0.5 \mathrm{ps}$ pulses). For the beginning of operation, the PETAL energy will be at the $1 \mathrm{~kJ}$ level, corresponding to intensity on target of $\sim 10^{20} \mathrm{~W} \mathrm{~cm}^{-2}$. The pulse duration can be varied between $0.5 \mathrm{ps}$ and $10 \mathrm{ps}$, and the intensity contrast is $10^{-7}$ at $-7 \mathrm{ps}$. Updates on the design and construction of the PETAL laser were given in 2011 2. The initial configuration of the LMJ/PETAL laser system is shown in Fig. 1

The development of the system has been funded by the Aquitaine Regional Council, with contribution from the French government and the EU (for a total budget 54.3 M€). The Aquitaine Regional Council is then the contracting owner of the PETAL facility while CEA is the prime contractor for its construction. Technical and scientific assistance are provided by ILP (Institut Lasers et Plasmas). Finally, PETAL is also considered as the major French contribution to the HiPER (High Power laser Energy Research facility) project, since it will allow to perform: i) significant experiments in the domain of fast ignition (allowing to produce hundreds of Joules of fast electrons); ii) backlighting for implosion experiments, in particular for direct-drive experiments on shock ignition (selected by HiPER as the main route to Inertial Fusion Energy).

The PETAL system is based on the Chirped Pulse Amplification technique. A short pulse laser oscillator (providing a bandwidth of $16 \mathrm{~nm}$ ) and an Offner stretcher (allowing bringing the pulse duration from $100 \mathrm{fs}$ to $9 \mathrm{~ns}$ ) form the front end. The preamplifier module (PAM) is based on the Optical Parametric Chirped Pulse Amplification (OPCPA) technique, and it produces pulses of $4.5 \mathrm{~ns}, 8 \mathrm{~nm}$, and $100 \mathrm{~mJ}$. The amplifier section produces pulses of $1.7 \mathrm{~ns}, 3 \mathrm{~nm}, 6.4 \mathrm{~kJ}$. The system is equipped with wavefront correction and chromatism correction.

The compression section includes 2 stages (in air and in vacuum, respectively). Finally the transport/focusing section includes a beam transport in vacuum to the LMJ interaction chamber. The focusing parabola (90 off-axis parabola) and the final pointing mirror are placed just outside the LMJ chamber. Also, the possibility of conversion of the PETAL pulse into the second harmonic has been taken into account (leaving the space for a conversion crystal) although this will not be implemented in the initial phase.

\section{Problems related Activation AND EMP}

Using a very high-energy high-intensity system like PETAL implies facing novel problems related to the chamber activation, and to the generation of giant electromagnetic pulses (EMP).

Activation of the experimental chamber and adjacent structures is due to the high-energy particles $(\gamma, \mathrm{p}, \mathrm{n})$ produced in experiments on PETAL coupled with LMJ. Several sources of these high energy particles are identified: 

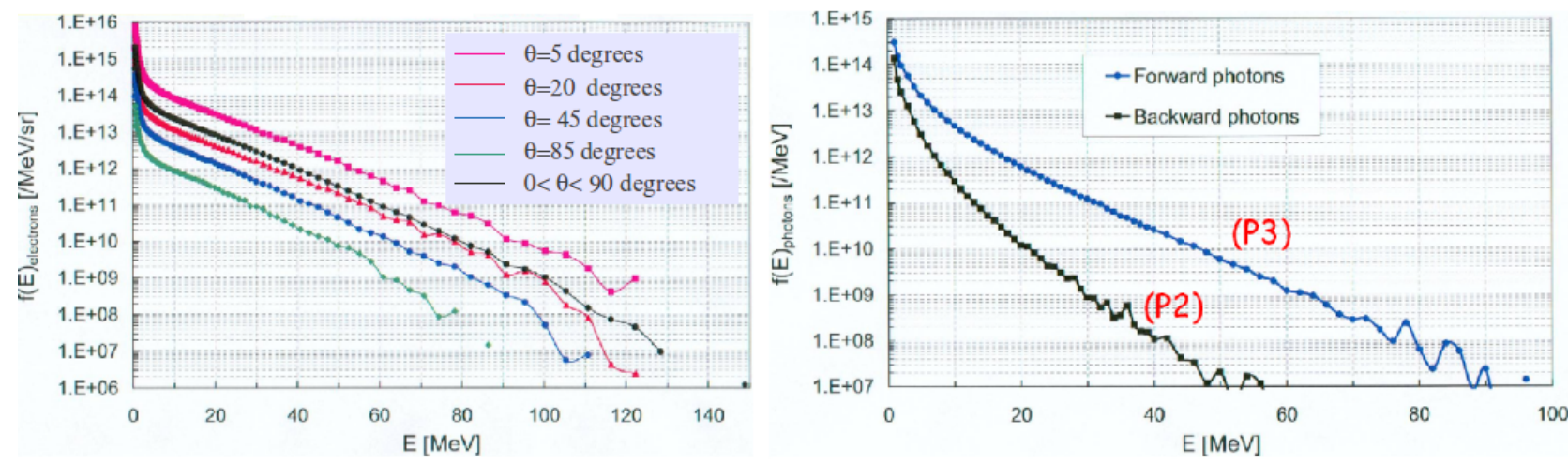

Figure 2. Fast electron source produced in interaction of the PETAL laser beam at $10^{20} \mathrm{~W} \mathrm{~cm}^{-2}$ with a plastic target (CALDER results at the end of the simulation, $t=2.2 \mathrm{ps}$ ) and corresponding photon distribution emitted from a $2 \mathrm{~mm} \mathrm{~W}$ target in the forward (P3) and backward (P2) directions (MCPNX results).

(1.) Laser interaction with thick solid targets and production of hard $\mathrm{X}$-rays and $\gamma$-rays.

(2.) Laser interaction with a thin solid target and production of high-energy protons and ions.

(3.) Laser interaction with compressed heavy hydrogen isotope targets (DD or DT in fast ignition experiments) and production of fusion neutrons (in the future).

A working group of specialists is now assessing the impact of the first two sources, with respect both to safety regulations and health risks (the third source will be considered in the future, when nuclear implosion experiments will be prepared).

The first type of radiation (hard X-rays and $\gamma$-rays) is caused by the fact that ultra-high-intensity interaction between laser and matter produces strong fluxes of relativistic electrons. When the target is thick (and especially if it is made of a high- $Z$ material) such electrons are stopped inside it and hard X-rays and $\gamma$-rays are produced by bremsstrahlung. A thick solid target then acts as a converter of fast electron energy into photon energy.

The first step of the work hence consists in modeling of the fast electron source. This has been done using the Particle-In-Cell (PIC) code CALDER 13 5. It describes the laser plasma interaction, electron acceleration and their transport through the target. However, generation of photons is not accounted for in this code because of a very large difference in the spatial and temporal scales. This process is considered separately in simulations performed with the code MCPNX [7, where a thick $(2 \mathrm{~mm})$ and large tungsten $(\mathrm{W})$ target is considered. From a simulation point of view, the electron source is defined at the edges of a (small) CALDER box and they are injected in a much larger MCNPX box. MCNPX then provides the number, direction and energy of the produced photons and secondary electrons, which are then responsible for the irradiation dose either directly or in photo-neutron reactions. About $10^{11}$ photo-neutrons are produced with a rather isotropic distribution and an average energy $\langle E\rangle=1.6 \mathrm{MeV}$.

Calculations show that the dose delivered by photons can be as high as 32 rads at $1 \mathrm{~m}$ at the rear of a W-target. In addition the activation zone is limited by a cone with an opening angle of $\sim 40^{\circ}$ around the laser beam axis. About $90 \%$ of the dose is provided by photons with energy $<23 \mathrm{MeV}$.

Figure 2 shows an example of the fast electron source obtained from CALDER simulations and of the corresponding photon source obtained from MCPNX simulations.

The second type of radiation (protons and ions) is produced in the process of the target normal sheath acceleration mechanism (TNSA) from the rear side of a thin target [27. On the LMJ/PETAL installation, the PW laser may be used for radiography of the plasma produced by ns LMJ beams. In that case the PETAL beam is focused on a secondary target, where a short $(\sim 20 \mathrm{ps})$ bunch of particles (electrons, protons, ions) is produced and directed to the primary plasma. The calculations of a proton source were performed with the PICLS two-dimensional (2D) PIC code [23]. The energy spectra and the angular divergences of the protons to be produced with PETAL are presented in Fig. 3. With a laser energy of $3.5 \mathrm{~kJ}$ in the PETAL beam we expect protons with energies up to $\sim 100 \mathrm{MeV}$, while at $1 \mathrm{~kJ}$ the expected cut-off is $\sim 40 \mathrm{MeV}$. (This number is compatible with $50 \mathrm{MeV}$ obtained on Omega EP by K. Flippo et al. 8 with $1 \mathrm{~kJ}$ but a longer pulse duration). The results concerning radiation doses are in agreement with those obtained in other laser facilities, and reported in Fig. 4. For instance, experimental data obtained at the Institute of Laser Engineering of Osaka University, using the LFEX laser (500 J shot at $10^{20} \mathrm{~W} \mathrm{~cm}^{-2}$ ) show a dose of $10 \mathrm{mSv}$ produced inside the interaction chamber [21, which is rather compatible with the dose of 32 rads given here. In comparison with existing lasers, due to its high energy and intensity, PETAL is expected to yield larger doses, see Fig. 4. 

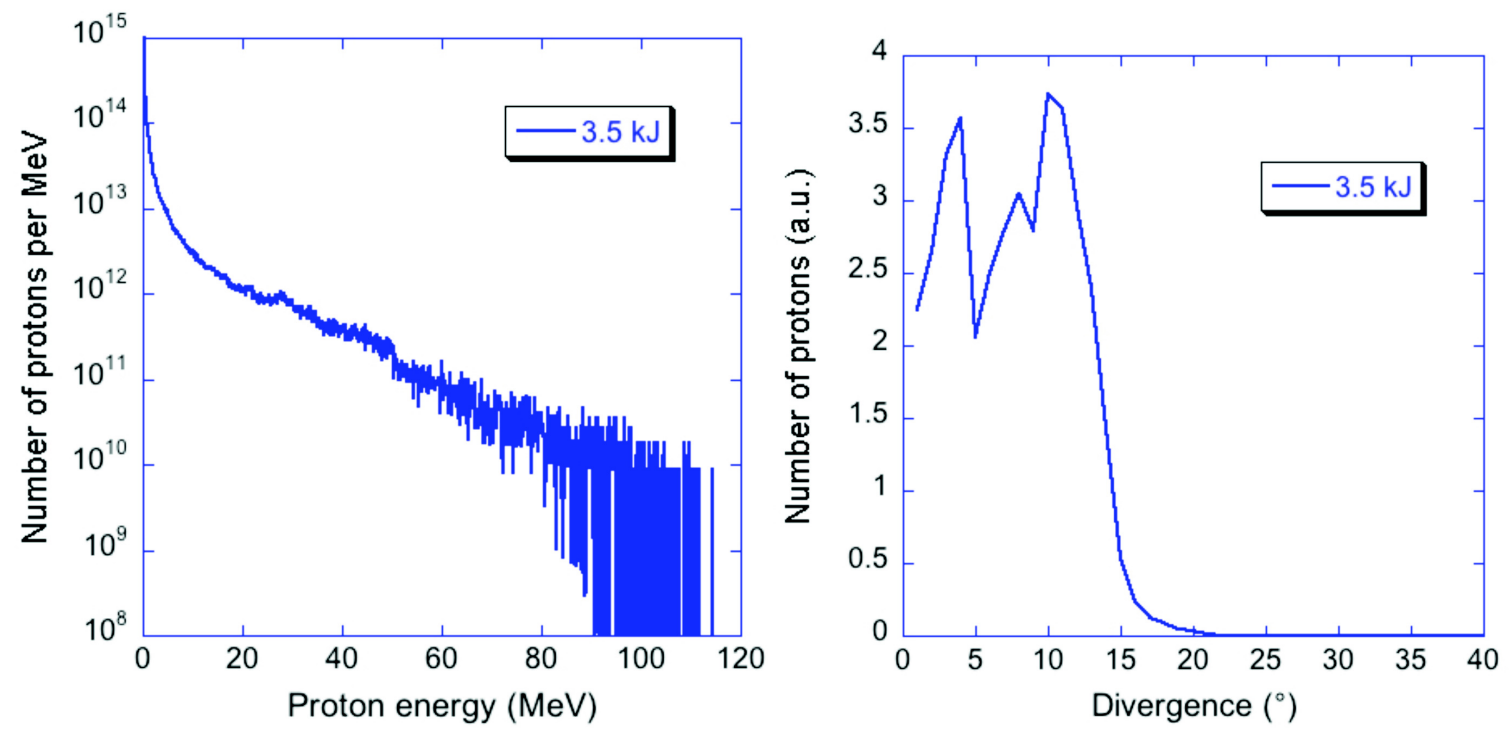

FiguRE 3. Left: expected spectrum of proton emission from a plastic $10 \mu \mathrm{m}$ target irradiated with PETAL at $3.5 \mathrm{~kJ}$ from PIC simulations using the code PICLS. Right: angular distribution of protons with an energy over $40 \mathrm{MeV}$ in the forward direction.

A second working group has been established to evaluate the problem of giant electromagnetic pulses (EMP) that are produced by:

(1.) Magnetic fields due to relativistic current propagating in solid targets.

(2.) Magnetic fields in plasma generated due to the crossed pressure and temperature gradients.

(3.) Electric and magnetic fields generated by fast electrons escaping the target.

(4.) Emission of the electrically charged target after the end of the laser pulse.

The first three these mechanisms produce very large amplitude fields which are located essentially inside the plasma. Their life time is limited to a few hundred ps at most. The last one is more dangerous for the diagnostic and control equipment as the corresponding fields are of a much longer duration (up to a $\mu$ s scale) and they fill all the chamber and escape outside through the diagnostic windows.

For instance, in experiments performed on the Omega laser facility in the US, in May 2011, using the Omega EP beam (1 kJ, $10 \mathrm{ps)}$ as a backlighter, an electric field as high as $\sim 250 \mathrm{kV} \mathrm{m}^{-1}$ was measured inside the interaction chamber. Such a field may affect the performance of diagnostics placed near the Target Chamber Centre (TCC). This field is reduced to $\sim 7.5 \mathrm{kV} \mathrm{m}^{-1}$ outside the interaction chamber. Fields as large as $\sim 750 \mathrm{~V} \mathrm{~m}^{-1}$ and $\sim 100 \mathrm{~V} \mathrm{~m}^{-1}$ were measured in the laser and diagnostic bays, respectively, at the distance of a few tens of meters. Results obtained in several laser facilities are shown in Fig. 5. It is clear that EMP features (both the amplitude of the field and the spectrum) depend on the application, i.e. the laser power/intensity, the target, the focal spot size, etc. In general radiographic (backlighting) applications imply a stronger EMP signal. We expect that the PETAL ps shots will produce more electric perturbations than the LMJ ns shots (typically for the ICF applications the EMP is limited to $\sim 100 \mathrm{kV} \mathrm{m}^{-1}$ mainly in the $1 \mathrm{GHz}$ range, while for backlighting a signal of the amplitude of $1 \mathrm{MV} \mathrm{m}^{-1}$ in the range of $10 \mathrm{GHz}$ is expected).

Coupling of such large EM field to electric cables may induce disturbances in the data acquisition electronics. The electromagnetic protection heavily affects the design of diagnostics tools (see next section) implying the need for reliable shielding and grounding of existing electronic equipment and design of new EMP-resistant electronics. In the meanwhile, in order to limit the problem, the choice in diagnostics mainly relies on passive detectors.

\section{DeVElopment of DiAgnostics}

The development of diagnostic tools is as essential as the construction of laser system itself. Only under this condition significant experiments in plasma physics could be performed. The first goal of the PETAL+ project is to develop detectors to characterize the emission of particles and radiation from targets irradiated with PETAL (so called "secondary sources"): energy range and spectrum, angular distributions and intensity. This is a specific project funded by the ANR (the French National Agency for Research) and managed by the University of Bordeaux, with a budget of $9.3 \mathrm{M} €$ (of which $1.3 \mathrm{M} €$ for the phase of exploitation of diagnostics and realization of experiments).

The cost of diagnostics is predicted to be much larger than for similar ones on smaller laser facilities. This is due to the fact that realization of di- 
agnostics must take into account the safety issues (chamber activation, radiation hazards, EMP, ...) as well as the need for remote handling \& remote control and access. The passive detectors used in the diagnostics (films, IPs, ... ) will need to be automatically extracted from the insertion ports and probably automatically processed.

The diagnostics themselves will be inserted in SID (Diagnostics insertion system) that will be moved in the interaction chamber and aligned to the target. In particular two SIDs will be used for PETAL+ diagnostics. They will be positioned almost in face of the PETAL beam entrance window to the LMJ interaction chamber (windows N.12 and 26).

Two working groups have been established to develop and design the diagnostics and SID for i) electron spectroscopy, proton spectroscopy and imaging, and ii) large-band X-ray spectroscopy. Because of the particular characteristics of the PETAL beam, large dynamical ranges have to be covered by these diagnostics. Other diagnostics will be installed in the future on the LMJ/PETAL facility.

The PETAL electron source spectrum is shown Fig. 2. It has been computed by E. Lefebvre and A. Compant La Fontaine [12 for radioprotection purposes. These calculations can be considered as majoring the intensities and electron energy spectra endpoints for the diagnostics design. Our diagnostics must cover the range $0.1 \div 150 \mathrm{MeV}$ for the protons and electrons. In addition to that, a spectrometer dedicated to the highest energy electrons (above $200 \mathrm{MeV}$ ) may be added. The energy resolution of the detectors will be of $5 \div 10 \%$ for both types of particles. The angular range to be covered by the diagnostics will be as large as $\sim 20^{\circ}$ around the direction normal to the PETAL target (protons/ions) or the incoming PETAL laser direction (electrons) to cover the full angular distributions.

The first aim of the PETAL + diagnostics is to characterize the particle emission from laser target interaction. To do so, electron magnetic spectrometers will be built in order to detect different parts of the spectrum: low, average and high energies. In addition to this, an activation detector such as the one developed by CENBG [10], which permits to measure the angular distribution of energetic electrons may be used. A small positron spectrometer is also under investigation within the PETAL+ project. The protons/ions will be detected with a two-component diagnostic. The first component will comprise a stack of radiochromic films or image plates, close to the target chamber center in order to cover the expected $20^{\circ}$ half-divergence of the proton/ion bunch. Stacking of these passive detection systems will permit to explore with a sufficient energy resolution the ion beam divergence. The second component will be a Thomson parabola 25. Such a detector, which combines the magnetic and electrostatic analysis of a bunch of particles will provide a clear separation between

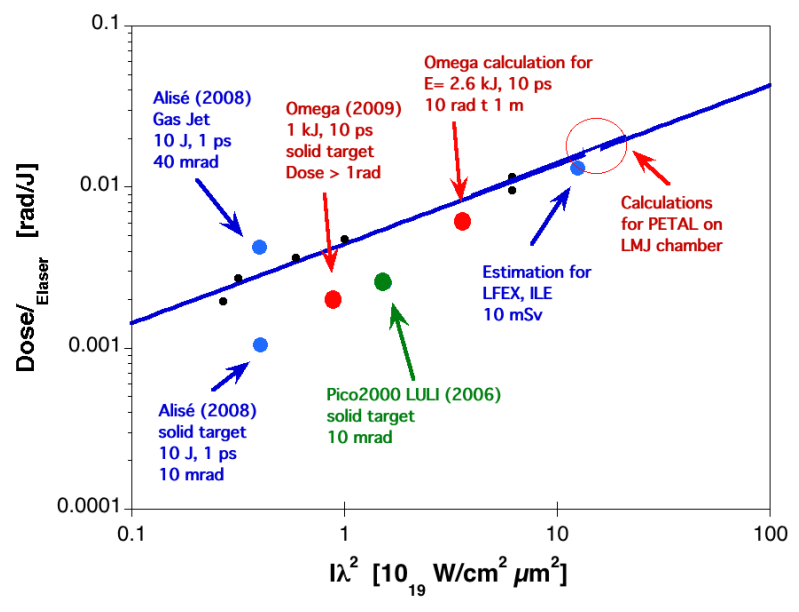

FiguRE 4. Radiation doses measured on several laser installations and comparison (solid line) with simulation results (calculated at $1 \mathrm{~m}$ behind a $2 \mathrm{~mm} \mathrm{~W}$ target).

the different ion species as well as good energy resolution needed to determine precisely the energy spectrum of the particles generated by the PW laser [9, 4]. In the present designs, this Thomson parabola will be used also for the magnetic analysis of electrons in the energy range $1 \div 150 \mathrm{MeV}$. After a detailed characterization of particle emission from PETAL targets, the diagnostics will be used for plasma experiments. As an example, we mention the proton radiography to determine the magnetic [14] or electric field [1] structure at the plasma scale or to measure the density of the LMJ plasmas [22, 26]. Stacks of radiochromic films and/or IPs will be used for the X-ray or proton radiography of targets irradiated with LMJ beams. The X-ray spectrometer, will have the Cauchois geometry [3, 6] and will cover the range $5 \div 100 \mathrm{keV}$. Such geometry, based on transmission cylindrical crystal, has been already adopted in many laboratories (HXS, HENEX, DCS at Naval Research Laboratory, TCS at Omega EP, LLCS at LLNL, LCS at LULI, C2S at CELIA) because of a potentially large spectral range which, in particular, allows to detect $K_{\alpha}$ emission lines from most materials with a variable resolution $\lambda / \Delta \lambda \sim 50 \div 300$, depending on the distance of the detector to the Roland circle.

Also the range $5 \div 100 \mathrm{keV}$ was chosen in order to complete the range of X-rays spectrometers already planned and under construction for the LMJ. This spectrometer is aiming at measuring the $K_{\alpha}$ lines of any materials. Such lines are in particular useful for fast ignition and shock ignition dedicated experiments since they act as tracer layers for the passage of fast electrons inside targets [24, 18]. 


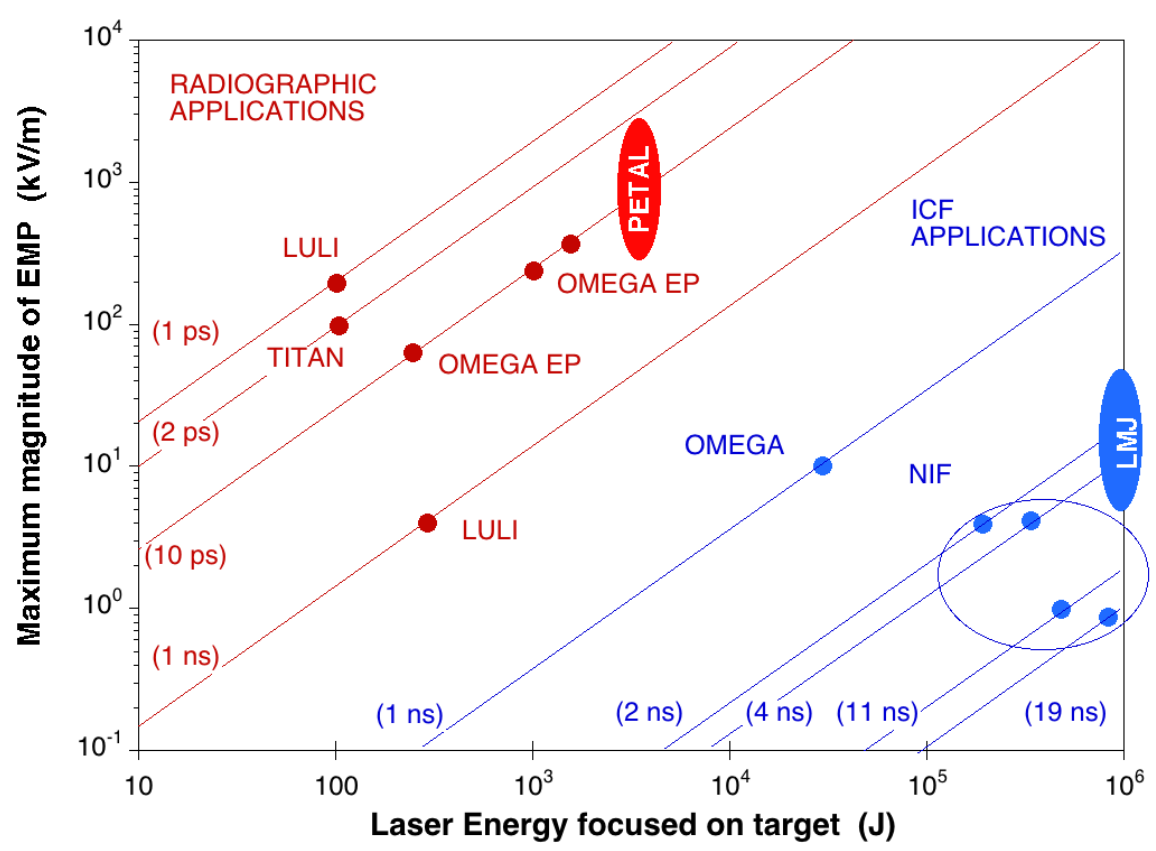

FigURE 5. EMP measurements performed by CEA on laser facilities: 2000-2004 LLE-Omega; 2006 LULI2000; 2007-2009 LULI-Pico2000; 2009-2010 LLE; in addition Omega EP US feedback on NIF and EMP measurements on TITAN National Ignition Campaign.

\section{PETAL IN THE CONTEXT OF EUROPEAN RESEARCH, DEVELOPMENT OF THE SCIENTIFIC RESEARCH PROGRAMME ON PETAL/LMJ}

Starting in 2015, the European scientific community will have access to PETAL/LMJ (with a contractual 20 to $30 \%$ of laser shots available for academic civilian research).

This will be a unique facility (the only other one being NIF) for addressing HED physics and Inertial Fusion in particular. PETAL is a key element of the HiPER project because i) it guarantees academic access to the LMJ/PETAL installations (through the agreement between CEA and the Region Aquitaine), ii) it will allow for integrated experiment in the domain of Fast Ignition (allowing to inject up to several hundred Joules of fast electrons into the target), iii) it will allow probing of LMJ implosion in integrated experiments related to Shock Ignition. However the installation itself will be very complex (problems of activation, of remote control, of EMP, ... ) and experiments must be carefully planned and prepared by using numerical simulations, targets and diagnostics. Preliminary experiments on smaller ("intermediate") laser facilities will be needed as an indispensable step before "final" experiments on LMJ/PETAL. For this reason, all the intermediate laser facilities are playing an essential role in the IFE research (Orion and Vulcan in the UK, LIL and LULI2000 in France, PALS in Prague, Phelix at GSI). These installations are also important from a strategic point of view as means to create the links between academic re- search, and training to research on very large laser systems.

The first phase of PETAL commissioning (starting from 2015) will include characterization of laser systems and diagnostics, studies of protons, X-rays, and electrons produced in laser plasma interactions. The second phase (from 2016) will be dedicated to physics experiments on extreme states of matter produced by short-pulse heating of solid targets.

The use of a PW laser such as PETAL within the context of plasma experiments provides basically two possibilities: either to heat isochorically a matter (creating WDM and HED states) or to create a beam of secondary energetic particles to probe the properties of plasma produced by the LMJ beams. In that second case, the high power of PETAL allows to generate intense beams of X-rays, gamma-rays, electrons and ions. They will allow to probe dense states created with shock or adiabatic compression of samples with LMJ beams. In particular, direct measurements of density, of the shock and fluid velocities can be made by using the proton and hard X-ray radiography. It will also be possible to use proton and hard X-ray backlighting to probe implosion and uniformity compression of targets imploded by LMJ (Shock Ignition approach to ICF, Polar Direct Drive).

Finally proton radiography could be used to measure magnetic fields, especially associated with jet formation in the domain of laboratory astrophysics.

Working groups are elaborating now a scientific program for the years to come for academic civilian research on LMJ/PETAL. This will be based on four "pillars": 


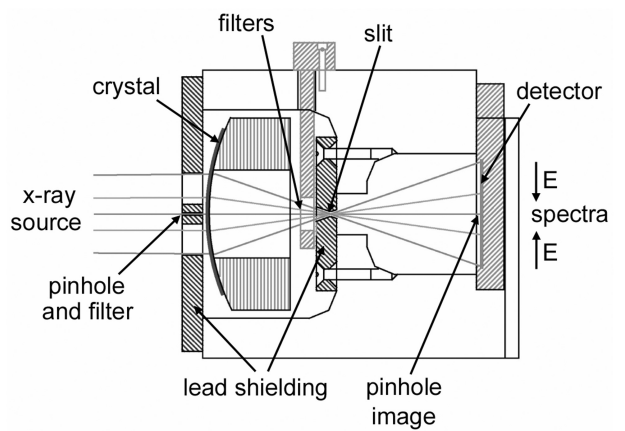

Figure 6. Scheme of a Cauchois transmission X-ray spectrometer

(1.) Fusion-opportunities for HiPER project: this will be a full-scale facility for demonstration of shock ignition of fusion targets.

(2.) Studies of matter in extreme conditions \& High Energy Density Physics.

(3.) Laboratory Astrophysics Experiments.

(4.) Acceleration and High Energy Physics.

Such a scientific program will define the scientific objective and priorities to be pursued on the installation. This programme will be validated by the ILP direction and by the international Scientific Advisory Committee of PETAL (SAC-P). An access to the PETAL/LMJ installation will be decided by the SAC-P on the basis of priorities indicated in the Scientific Programme. A call for experimental proposals will be announced as soon as the installation will be operational. To facilitate the access an "users' committee" (e.g. similar to the one operating at Omega facility) will be established.

\section{REFERENCES}

[1] D. Batani, et al. LASER-driven fast electron dynamics in gaseous media under the influence of large electric fields. Physics of Plasmas 16:033104, 2009.

[2] N. Blanchot. Overview of PETAL, the multi-petawatt project in the LMJ facility. IFSA 11 conference, September \& Workshop on the physics with PETAL, December, Bordeaux, France, 2011.

[3] Y. Cauchois. Spectrographie des rayons x par transmission d'un faisceau non canalisé à travers un cristal courbé. Journal de Physique 3:320, 1932.

[4] Jung D., et al. Development of a high resolution and high dispersion Thomson parabola. Review of Scientific Instruments 82:013306, 2011.

[5] E. d'Humières, et al. Proton acceleration mechanisms in high-intensity laser interaction with thin foils. Physics of Plasmas 12:062704, 2005.

[6] Seely J. F., et al. Hard X-ray spectroscopy of inner-shell $\mathrm{K}$ transitions generated by $\mathrm{MeV}$ electron propagation from intense picosecond laser focal spots. High Energy Density Physics 3:263, 2007.

[7] M. L. Fensin, J. S. Hendricks, S. Anghaie. The enhancements and testing for the MCNPX 2.6.0 depletion capability. Journal of Nuclear Technology 170:68-79, 2010. Based on LA-UR-08-0305.
[8] K. Flippo, et al. Measurements of proton generation with intense kilojoule laser. Journal of Physics: Confence Series 244:022033, 2010.

[9] C. G. Freeman, et al. Calibration of a Thomson parabola ion spectrometer and Fujifilm imaging plate detectors for protons, deuterons, and alpha particles. Review of Scientific Instruments 82:073301, 2011.

[10] M. Gerbaux, et al. High flux of relativistic electrons produced in femtosecond laser-thin foil target interactions: Characterization with nuclear techniques. Review of Scientific Instruments 79:023504, 2008.

[11] S. H. Glenzer, et al. Demonstration of ignition radiation temperatures in indirect-drive inertial confinement fusion hohlraums. Physical Review Letters 106:085004 \& 109903, 2011.

[12] E. Lefèbvre, A. Compant La Fontaine. Note de calculs termes sources pour petal couple a la chambre $\operatorname{lmj}$, 2011. CEA-DIF.

[13] E Lefèbvre, et al. Electron and photon production from relativistic laser-plasma interactions. Nuclear Fusion 3:629, 2003.

[14] C. K. Li, et al. Observations of electromagnetic fields and plasma flow in hohlraums with proton radiography. Physical Review Letters 102:205001, 2009.

[15] J. D. Lindl. The national ignition campaign: goals and progress. IFSA11 conference, Bordeaux, France, 2011 and references therein.

[16] C. Lion. The LMJ program: an overview. IFSA11 conference, Bordeaux, France, 2011.

[17] E. Martinolli, et al. Conical crystal spectrograph for high brightness X-ray $k_{\alpha}$ spectroscopy in subpicosecond laser-solid interaction. Review Scientific Instruments 5:2024, 2004.

[18] E. Martinolli, et al. Fast electron transport and heating of solid targets in high intensity laser interaction measured by $k_{\alpha}$ fluorescence. Physical Review E 73:046402, 2006.

[19] J. L. Miquel. Present status of the LMJ facility. IFSA11 conference, Bordeaux, France, 2011.

[20] E. L. Moses. The NIF: an international high energy density science and inertial fusion user facility. IFSA11 conference, Bordeaux, France, 2011.

[21] Osaka Courtesy of Prof. H. Nishimura, ILE.

[22] J. R. Rygg, et al. Proton radiography of inertial fusion implosions. Science 319:1223, 2008.

[23] Y. Sentoku, A. Kemp. Hot-electron energy coupling in ultraintense laser-matter interaction. Journal of Computational Physics 227:6846, 2008.

[24] R. B. Stephens, et al. $k_{\alpha}$ fluorescence measurement of relativistic electron transport in the context of fast ignition. Physical Review E 69:066414, 2004.

[25] J. J. Thomson. Rays of positive electricity. Philosophical Magazine 21:225, 1911.

[26] L. Volpe, et al. Proton radiography of laser-driven imploding target in cylindrical geometry. Physics of Plasmas 18:012704, 2011.

[27] S. Wilks, et al. Energetic proton generation in ultra-intense laser-solid interactions. Physics of Plasmas 8:542, 2001. 